\title{
The Influence of Yb Substitution on the Magnetic, Electric Properties and Electronic Structure of $\mathrm{Yb}_{x} \mathrm{Gd}_{1-x} \mathrm{Ni}_{5}$ System
}

\author{
A. BAJOREK ${ }^{a}$ And G. CheŁkOWska ${ }^{a}$ \\ A. Chełkowski Institute of Physics, University of Silesia \\ Uniwersytecka 4, 40-007 Katowice, Poland
}

\begin{abstract}
The crystal and electronic structure, magnetic and electric properties of intermetallic compounds $\mathrm{Yb}_{x} \mathrm{Gd}_{1-x} \mathrm{Ni}_{5}(x=0.0,0.2,0.4,0.5)$ are presented. The whole series crystallize in the hexagonal $\mathrm{CaCu}_{5}$ type of crystal structure. The ordering temperatures $\mathrm{T}_{\mathrm{C}}$ and the effective magnetic moments decrease with increase in ytterbium concentration. The electronic structure studied by X-ray photelectron spectroscopy method shows the domination of $\mathrm{Ni} 3 d$ states nearby the Fermi level. The multiplet structure visible in valence bands is typical of $\mathrm{Yb}^{3+}$ ions.
\end{abstract}

PACS numbers: 71.20.Eh, 75.30.Cr, 79.60.-i

\section{Introduction}

The $\mathrm{RT}_{5}$ compounds, where $\mathrm{R}$ is rare earth and $\mathrm{T}$ is a transition metal, show very interesting magnetic properties due to the combination of $3 d$ itinerant and $4 f$ localized magnetism [1-11]. One of the most known series is $\mathrm{RNi}_{5}$. The analysis of its magnetic properties shows that the Curie temperatures are very low. The highest value of $T_{\mathrm{C}}$ was evidenced for $\mathrm{GdNi}_{5}$ compound [1-4] which was obtained for the first time in 1962 by Nesbitt et al. [6]. Latter studies showed that $\mathrm{GdNi}_{5}$ is the ferrimagnet below $T_{\mathrm{C}}=32 \mathrm{~K}$ and the value of magnetization extrapolated to $0 \mathrm{~K}$ equals $6.2 \mu_{\mathrm{B}}$ /f.u. $[4,7,11]$. This kind of behavior is connected with a negative polarization of the $\mathrm{Ni} 3 d$ band by $\mathrm{Gd}$ atoms. The $\mathrm{RT}_{5}$ compounds where $\mathrm{R}=\mathrm{Yb}$ are very interesting due to possible existence of the intermediate valence state. The $\mathrm{Yb}$ ions in $\mathrm{RT}_{5}$ systems often indicate the occurrence of the magnetic trivalent state $\mathrm{Yb}^{3+}$ with $4 f^{13}$ localized electrons, but also the non-magnetic divalent $\mathrm{Yb}^{2+}$ state with nonlocalized $4 f^{14}$ electrons or the intermediate valence state [8-10].

In this paper we present the influence of partially replacing of Gd by Yb atoms on the crystal structure, magnetic and electric properties as well as on the electronic structure in $\mathrm{Yb}_{x} \mathrm{Gd}_{1-x} \mathrm{Ni}_{5}$ system.

\section{Experimental}

Polycrystalline samples of the $\mathrm{Yb}_{x} \mathrm{Gd}_{1-x} \mathrm{Ni}_{5}$ series with $x=0.0,0.2,0.4,0.5$ were prepared by arc melting from high purity elements under argon atmosphere. The crystal structure of all samples was checked by means of X-ray diffraction using Siemens D5000 diffractometer. The ac magnetic susceptibility measurements were done in the temperature range $4.2-300 \mathrm{~K}$ at frequency $1 \mathrm{kHz}$. The dc susceptibility was measured in the paramagnetic range 300-800 K using the Faraday method in the magnetic field of $0.38 \mathrm{~T}$. The electrical resistance $R(T)$ was measured quasi-continuously at a slowly changing temperature $(4.2-300 \mathrm{~K})$ by a standard four-probe technique. The X-ray photoelectron spectroscopy (XPS) measurements were performed with the use of PHI 5700/660 Physical Electronics spectrometer. The spectra were analyzed at room temperature using monochromatized $\mathrm{Al} K_{\alpha}$ radiation $(1486.6 \mathrm{eV})$. The samples were fractured and measured in vacuum of $10^{-10}$ Torr.

\section{Results and discussion}

\subsection{Crystal structure}

The crystal structure of $\mathrm{Yb}_{x} \mathrm{Gd}_{1-x} \mathrm{Ni}_{5}$ samples was studied by X-ray powder diffraction (XRD). The analysis of XRD patterns obtained at room temperature confirms that all as-cast studied compounds crystallize in the hexagonal $\mathrm{CaCu}_{5}$ structure. As it is shown in Table, the volume $V$ of the unit cell decreases with rising $\mathrm{Yb}$ content up to $x=0.4$. In parallel, the $a$ lattice parameter decreases up to $x=0.4$, while $c$ parameter increases with a lower rate in the whole range of studied concentrations. This kind of behavior is probably connected with different ionic radii for gadolinium $\left(\mathrm{Gd}^{3+}-1.02 \AA\right)$ and ytterbium $\left(\mathrm{Yb}^{3+}-0.86 \AA\right)$. The anomalous expansion above $x=0.4$ may be related to the occurrence of an $\mathrm{Yb}$ intermediate valence. Similar behavior was previously observed for $\mathrm{YbCu}_{5-x} \mathrm{Al}_{x}$ series [8]. The decrease 
in $a$ and the increase in $c$ lattice parameters is possible in ternary compounds of $\mathrm{CaCu}_{5}$ structure due to their layer structure.

TABLE

The crystal structure parameters, magnetic and electric properties of $\mathrm{Yb}_{x} \mathrm{Gd}_{1-x} \mathrm{Ni}_{5}$ system.

\begin{tabular}{c|c|c|c|c|c|c|c}
\hline \hline$x$ & $\begin{array}{c}a \\
{[\AA]}\end{array}$ & $\begin{array}{c}c \\
{[\AA]}\end{array}$ & $\begin{array}{c}V \\
{\left[\AA^{3}\right]}\end{array}$ & $\begin{array}{c}T_{\mathrm{C}} \\
{[\mathrm{K}]}\end{array}$ & $\begin{array}{c}\mu_{\mathrm{eff}} \\
{\left[\mu_{\mathrm{B}} / \text { f.u. }\right]}\end{array}$ & $\begin{array}{c}\theta_{\mathrm{p}} \\
{[\mathrm{K}]}\end{array}$ & $\begin{array}{c}\rho_{0} \\
{[\mu \Omega \mathrm{cm}]}\end{array}$ \\
\hline 0.0 & 4.9120 & 3.9647 & 82.84 & 33.7 & 8.35 & 7.24 & 27.28 \\
0.2 & 4.8950 & 3.9727 & 82.44 & 24.3 & 8.07 & -16.7 & - \\
0.4 & 4.8807 & 3.9792 & 82.09 & 18.8 & 7.50 & -17.3 & 12.29 \\
0.5 & 4.8810 & 3.9857 & 82.23 & 13.3 & 7.14 & -34.6 & 9.25
\end{tabular}

\subsection{Magnetic properties}

The magnetic properties of $\mathrm{Yb}_{x} \mathrm{Gd}_{1-x} \mathrm{Ni}_{5}$ series were studied using the temperature dependence of $\chi_{\mathrm{ac}}(T)$ and $\chi_{\mathrm{dc}}(T)$ magnetic susceptibility. From the plots of $\chi_{\mathrm{ac}}(T)$ the values of the Curie temperature $T_{\mathrm{C}}$ were obtained. These values decrease from $33.7 \mathrm{~K}(x=0.0)$ to $13.3 \mathrm{~K}$ $(x=0.5)$. The same behavior was noticed for the effective magnetic moment $\left(\mu_{\mathrm{eff}}\right)$ obtained from $\chi_{\mathrm{dc}}(T)$ measurements. The values of $\mu_{\mathrm{eff}}$ deduced from the Curie constants decrease from $8.35 \mu_{\mathrm{B}} /$ f.u. $(x=0.0)$ to $7.14 \mu_{\mathrm{B}} /$ f.u. $(x=0.5)$. We can suppose that substitution of $\mathrm{Yb}$ with smaller effective moment $\left(\mathrm{Yb}^{3+}\right.$ $\left.4.54 \mu_{\mathrm{B}}\right)$ in the place of $\mathrm{Gd}$ atoms causes the reduction of the total value of $\mu_{\text {eff }}$. However, the fact that the value of $\mu_{\text {eff }}$ in $\mathrm{GdNi}_{5}$ is higher than for free $\mathrm{Gd}^{3+}$ ion may be correlated with spin fluctuations as it was previously reported for different 1:5 compounds e.g. by Coldea et al. $[3,5]$. With the substitution of $\mathrm{Gd}$ by $\mathrm{Yb}$ atoms the presence of the ferrimagnetic ordering was observed for all compounds, which was reflected in the negative values of paramagnetic temperatures (Table).

\subsection{Electrical resistivity}

The temperature dependence of electrical resistance $R(T)$ was measured for the whole $\mathrm{Yb}_{x} \mathrm{Gd}_{1-x} \mathrm{Ni}_{5}$ series. The values of the Curie temperature $T_{\mathrm{C}}$ obtained from the plots $R(T)$ were in good agreement with those derived from the magnetic measurements. Knowing the dimensions of samples we were able to obtain the thermal variation of electrical resistivity $\rho(T)$ besides the sample with $x=0.2$ which had a lot of microcracks. The $\rho(T)$ dependence for $\mathrm{Yb}_{x} \mathrm{Gd}_{1-x} \mathrm{Ni}_{5}$ series shows a typical metallic behavior. The value of residual resistivity obtained experimentally at $4.2 \mathrm{~K}$ decreased with increase in $\mathrm{Yb}$ concentration from $\rho_{0}=27.28 \mu \Omega \mathrm{cm}(x=0.0)$ to $\rho_{0}=9.25 \mu \Omega \mathrm{cm}(x=0.5)$ (Table). The highest value of $\rho_{0}$ for $\mathrm{GdNi}_{5}$ compound may be correlated with the microcracks which were observed for this compound. The decrease in $\rho_{0}$ with increase in $\mathrm{Yb}$ concentration is connected with different sizes of ionic radii for $\mathrm{Yb}^{3+}(0.94 \AA)$ and $\mathrm{Gd}^{3+}(1.02 \AA)$ ions. This kind of behavior can suggest that $\mathrm{Yb}$ ions occur in trivalent state because the value of ionic radius for $\mathrm{Yb}^{3+}(0.94 \AA)$ is smaller than $\mathrm{Yb}^{2+}(1.13 \AA)$.

\subsection{Electronic structure}

The valence band (VB) spectra for $\mathrm{Yb}_{x} \mathrm{Gd}_{1-x} \mathrm{Ni}_{5}$ series in the energy range of $2 \div-12 \mathrm{eV}$ are presented in Fig. 1. The shape of VB spectra nearby the Fermi level $\left(E_{\mathrm{F}}\right)$ observed for $\mathrm{Yb}_{x} \mathrm{Gd}_{1-x} \mathrm{Ni}_{5}$ series is determined for

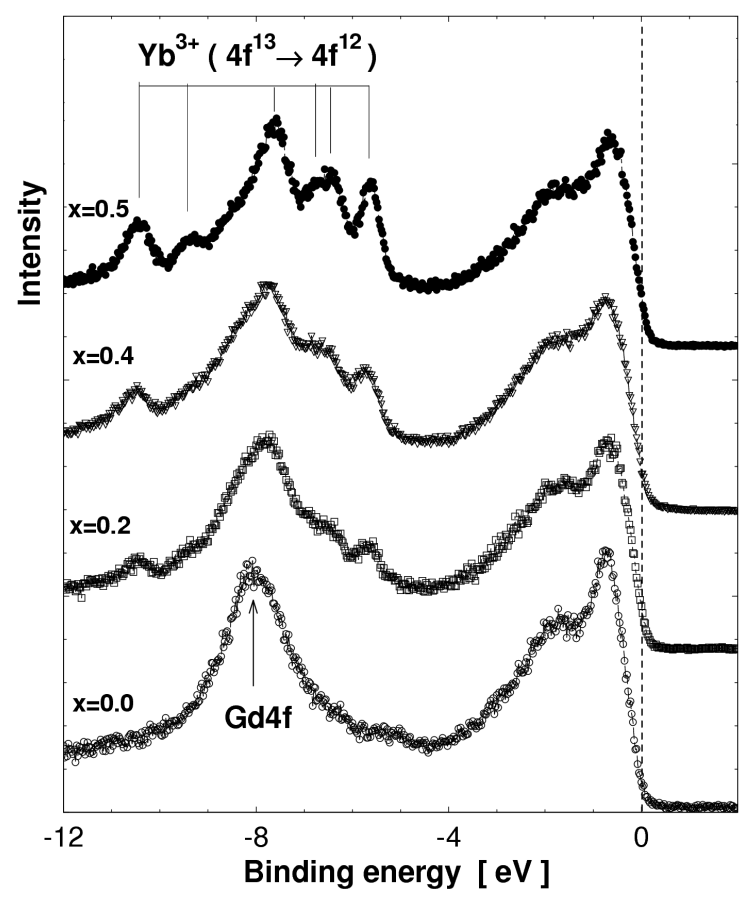

Fig. 1. The valence band spectra of $\mathrm{Yb}_{x} \mathrm{Gd}_{1-x} \mathrm{Ni}_{5}$ series in the energy range of $2 \div-12 \mathrm{eV}$.

$\mathrm{Ni} 3 d$ states. The substitution of $\mathrm{Yb}$ in the place of Gd causes the shift of the valence band into lower binding energies. These shifts reach the value of $0.1 \mathrm{eV}$ for $x=0.5$ and may be correlated with $d-f$ interaction as well as with the presence of a small amount of $\mathrm{Yb}^{2+}$ states. The similar shift about $0.1 \mathrm{eV}$ were also observed for the Ni $2 p$ core level lines. The multiplet structure visible at about $-5 \div-12 \mathrm{eV}$ binding energy range for compounds with $x \geq 0.2$ is mainly coming from trivalent $\mathrm{Yb} 4 f$ ions. It is connected with $4 f^{13} \longrightarrow 4 f^{12}$ transition and consists of six lines partly overlapping with $\mathrm{Gd}$ $4 f$. About $6 \mathrm{eV}$ below $E_{\mathrm{F}}$ one can observe small satellite structure related to two-hole nickel final states: $3 d^{9} 4 s$ (main line) and $3 d^{8} 4 s^{2}$ (satellite line). The analysis of Yb $4 d$ core level lines (Fig. 2) shows the presence of multiplet structure connected with the interaction with core hole $4 d$ and $4 f$ electrons. There are visible several lines at about $-184.5 \mathrm{eV},-192 \mathrm{eV}$ and $-199 \mathrm{eV}$ which are typical of $\mathrm{Yb}^{3+}$ states. In contrast to $\mathrm{YbXCu}_{4}$ compounds $[9,10]$ the core level $\mathrm{Yb} 4 d$ spectra for $\mathrm{Yb}_{x} \mathrm{Gd}_{1-x} \mathrm{Ni}_{5}$ 


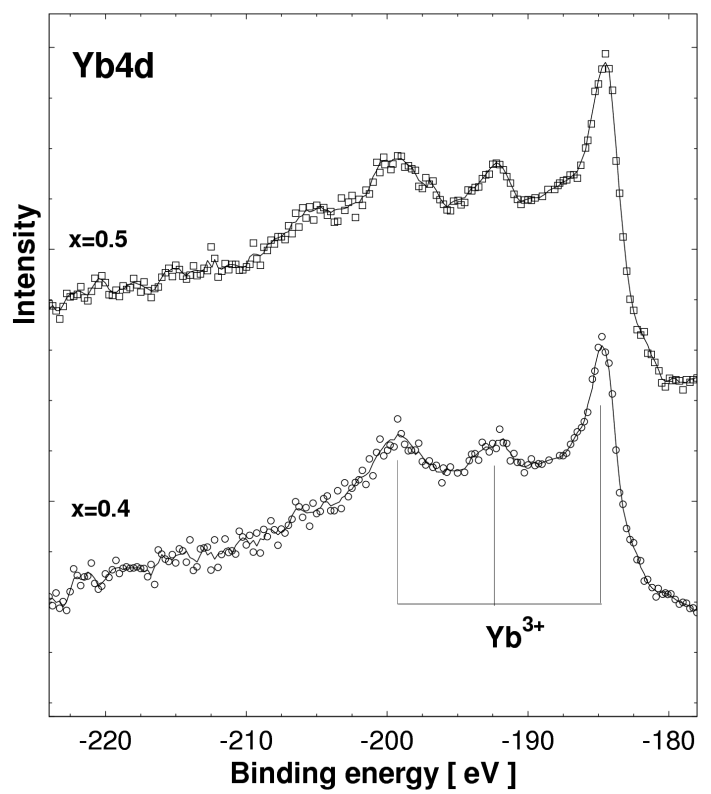

Fig. 2. The $\mathrm{Yb} 4 d$ core level lines for $\mathrm{Yb}_{0.4} \mathrm{Gd}_{0.6} \mathrm{Ni}_{5}$ and $\mathrm{Yb}_{0.5} \mathrm{Gd}_{0.5} \mathrm{Ni}_{5}$ compounds.

series do not show clearly the presence of ytterbium divalent states. For $\mathrm{YbXCu}_{4}$ compounds the peaks typical of these states were observed at about $-181.5 \mathrm{eV}$ and $-191 \mathrm{eV}$. We do not observe the first peak $(-181.5 \mathrm{eV})$ which is typical of $\mathrm{Yb}^{2+}$ ions. In the Ni $2 p$ spectra (not shown here) the satellite structures at about $6 \mathrm{eV}$ above the main lines are visible for all investigated compounds. The presence of the satellites in this energy range is typical of the spectrum of pure Ni element with not fully filled $3 d$ band and suggests that nickel atoms carry a magnetic moment, which is in agreement with band structure calculations for other $\mathrm{RNi}_{5}$ compounds [7, 11].

\section{Conclusions}

The influence of $\mathrm{Yb}$ substitution is reflected in the magnetic and electric properties as well as in the crystal and electronic structure in $\mathrm{Yb}_{x} \mathrm{Gd}_{1-x} \mathrm{Ni}_{5}(x \leq 0.5)$ compounds.

The substitution of $\mathrm{Gd} / \mathrm{Yb}$ causes the increase in $c(x)$ lattice parameter in the whole range of concentrations. This kind of change is connected with different ionic radii for gadolinium and yterrbium ions. The deviation from the linear change of $a(x)$ and $V(x)$ for $x>0.4$ may be related to intermediate valence of $\mathrm{Yb}$ ions.
The $\mathrm{Yb}$ substitution causes the decrease in $T_{\mathrm{C}}$ as well as the value of $\mu_{\text {eff }}$ obtained from the Curie constant. The gradual decrease in $\rho_{0}$ was observed due to trivalent state of $\mathrm{Yb}$ ions which have smaller ionic radius than $\mathrm{Yb}^{2+}$ ions.

The XPS valence band nearby the $E_{\mathrm{F}}$ for the whole series are dominated by Ni $3 d$ states. The multiplet structure visible in the valence bands is characteristic of $\mathrm{Yb}^{3+}$ states. With increase in $\mathrm{Yb}$ content there were observed small energy shifts into lower binding energies. It can be the result of $d-f$ interaction but also the presence of small amounts of $\mathrm{Yb}^{2+}$ states. The valence of ytterbium can be also estimated from $\mathrm{Yb} 4 d$ spectra. However, we suppose that the multiplet structure of $\mathrm{Yb} 4 d$ core level line is rather characteristic of $\mathrm{Yb}^{3+}$ structure.

\section{Acknowledgments}

This work was supported by funds for science in years 2007-2010 as a research project no. N202 134933.

\section{References}

[1] K.H.J. Buschow, Rep. Prog. Phys. 40, 1169 (1977).

[2] E. Burzo, A. Chełkowski, H.R. Kirchmayr, Landolt Börnstein Handbook, Vol. III/19d2, Springer, Berlin 1990.

[3] M. Coldea, S.G. Chiuzbaian, M. Neumann, D. Todoran, M. Demeter, R. Tetean, V. Pop, Acta Phys. Pol. A 98, 629 (2000).

[4] D. Gignoux, D. Givord, A. Del. Moral, Solid State Commun. 19, 891 (1976).

[5] E. Burzo, V. Pop, I. Costina, J. Magn. Magn. Mater. 157/158, 615 (1996).

[6] E.A. Nesbitt, H.J. Williams, J.H. Wernick, R.C. Sherwood, J. Appl. Phys. 33, 1674 (1962).

[7] A. Bajorek, D. Stysiak, G. Chełkowska, J. Deniszczyk, W. Borgieł, M. Neumann, Mater. Sci. (Poland) 24, 867 (2006).

[8] J. He, G. Ling, Z. Ye, J. Alloys Comp. 325, 54 (2001).

[9] H. Sato, K. Hiraoka, M. Taniguchi, Y. Nishikawa, F. Nagasaki, H. Fujino, Y. Takeda, M. Arita, K. Shimada, H. Namatame, A. Kimura, K. Kojima, J. Phys., Condens. Matter 14, 4445 (2002).

[10] H. Sato, Y. Nishikawa, F. Nagasaki, H. Fujino, Y. Takeda, M. Arita, K. Shimada, H. Namatame, A. Kimura, M. Taniguchi, K. Hiraoka, K. Kojima, Surf. Sci. Lett. 9, 1079 (2002).

[11] E. Burzo, L. Chioncel, I. Costina, S.G. Chiuzbaian, also J. Phys., Condens. Matter 18, 4861 (2006). 